\title{
ORIGINAL
}

\author{
A. Fereidoon • Y. Rostamiyan • M. Akbarzade • \\ Davood Domiri Ganji
}

\section{Application of He's homotopy perturbation method to nonlinear shock damper dynamics}

Received: 25 September 2008 / Accepted: 13 May 2009 / Published online: 28 May 2009

(C) The Author(s) 2009. This article is published with open access at Springerlink.com

\begin{abstract}
In order to obtain the equations of motion of vibratory systems, we will need a mathematical description of the forces and moments involved, as function of displacement or velocity, solution of vibration models to predict system behavior requires solution of differential equations, the differential equations based on linear model of the forces and moments are much easier to solve than the ones based on nonlinear models, but sometimes a nonlinear model is unavoidable, this is the case when a system is designed with nonlinear spring and nonlinear damping. Homotopy perturbation method is an effective method to find a solution of a nonlinear differential equation. In this method, a nonlinear complex differential equation is transformed to a series of linear and nonlinear parts, almost simpler differential equations. These sets of equations are then solved iteratively. Finally, a linear series of the solutions completes the answer if the convergence is maintained; homotopy perturbation method (HPM) is enhanced by a preliminary assumption. The idea is to keep the inherent stability of nonlinear dynamic; the enhanced HPM is used to solve the nonlinear shock absorber and spring equations.
\end{abstract}

Keywords Nonlinear damping · Nonlinear spring · Nonlinear differential equation · Enhanced HPM $($ EHPM $) \cdot$ He's homotopy perturbation method $\cdot$ Shock absorber

\section{Introduction}

A spring element exerts a reaction force in response to a displacement, either compression or extension, of the element. The linear spring relation, $f=k x$, become less accurate with increasing deflection (for either compression or extension). In such case it is often replaced by [1]:

$$
f=k_{1} x+k_{2} x^{3}
$$

where $k_{1}>0$ and has dimension of $N / m$ and $x$ is the spring displacement from its free length. The spring elements is said to be hardening or hard if $k_{2}>0$ and has dimension of $N / m^{3}$ and softening or soft if $k_{2}<0$. These cases are shown in Fig. 1.

The spring stiffness $k$ is the slope of force-deflection curve and is constant for the linear spring element. A nonlinear spring does not have a single stiffness value since its slope is variable.

\footnotetext{
A. Fereidoon

Department of Mechanical Engineering, Faculty of Engineering, Semnan University, Semnan, Iran

Y. Rostamiyan

Department of Mechanical Engineering, Islamic Azad University-Semnan Branch, Semnan, Iran

M. Akbarzade, D. D. Ganji ( $\varangle)$

Department of Mechanical Engineering, Babol University of Technology, P. O. Box 484, Babol, Iran

E-mail:ddg_davood@yahoo.com; mirgang@nit.ac.ir
} 


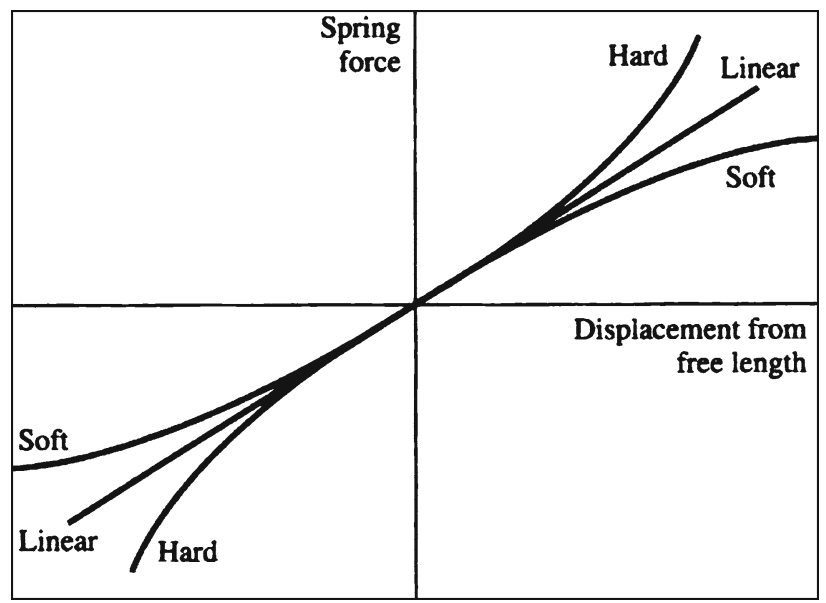

Fig. 1 Hard, soft and linear spring function [1]

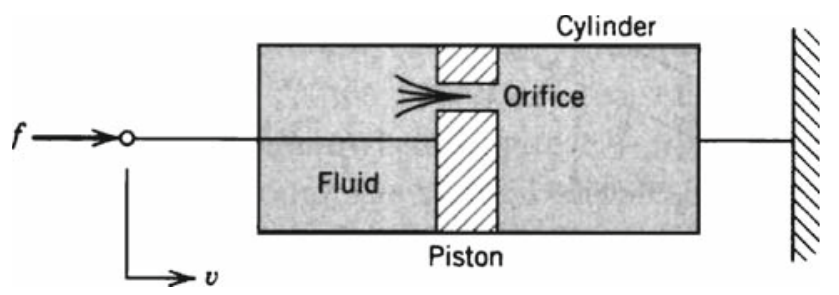

Fig. 2 A fluid damper consists of a piston moving inside a cylinder filled with a viscous fluid

A damper element is an element that resists relative velocity across it. A simple way to achieve damping is with a dashpot or damper, which is the basis of shock absorber. It consists of a piston moving inside a cylinder that is sealed and filled with a viscous fluid (Fig. 2) the piston has a hole or orifice through which the fluid can flow when the piston moves relative to the cylinder, but the fluid' viscosity resists this motion [1].

\section{Shock damper}

The telescopic shock damper is used in many vehicles. A cutaway view a typical shock damper is very complex, but the basic principle of its operation is the damper concept illustrated in Fig. 3. The damping can be designed to be progressive, which means that the slope of the damping force-versus-velocity curve increasing with velocity, or degressive, where the slope decreases with velocity (Fig. 4) [1].

\section{Homotopy perturbation method}

The widely applied techniques (i.e., perturbation method) are of great interest to be used in engineering systems $[2,3]$. To eliminate the limitation of "small parameter", which is assumed in the perturbation method, a new technique based on the homotopy terminology [4-6] has been proposed. Accordingly, a nonlinear problem is transformed into an infinite number of simple problems without using the perturbation technique. Effectively, letting the small parameter float and converge to the unity, the problem will be converted into a special perturbation problem with a small embedding parameter. So the method receives the name, homotopy perturbation method (HPM). The effectiveness of the new technique is presented in $[7,8]$. This approach can take full advantage of the traditional perturbation technique and homotopy analysis method. The new scheme has been applied to linear and nonlinear ordinary and partial differential equations. These equations usually describe a dynamic system incorporating the perturbation value (i.e., HPM). It has been applied to a wide class of differential equations, such as Duffing equation [2], numerical and algebraic methods $[4,9,10]$ and heat transfer [10]. To illustrate the basic ideas of this method, we consider the following equation [11-13]:

$$
A(u)-f(r)=0, \quad r \in \Omega,
$$




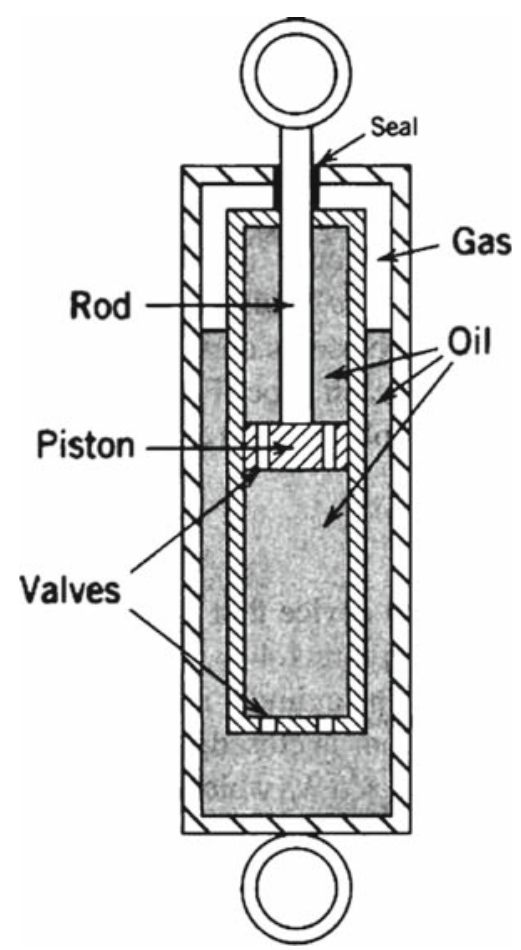

Fig. 3 A shock damper in common parlance (or damper in technical use) is a mechanical device designed to smooth out or damp shock impulse, and dissipate kinetic energy

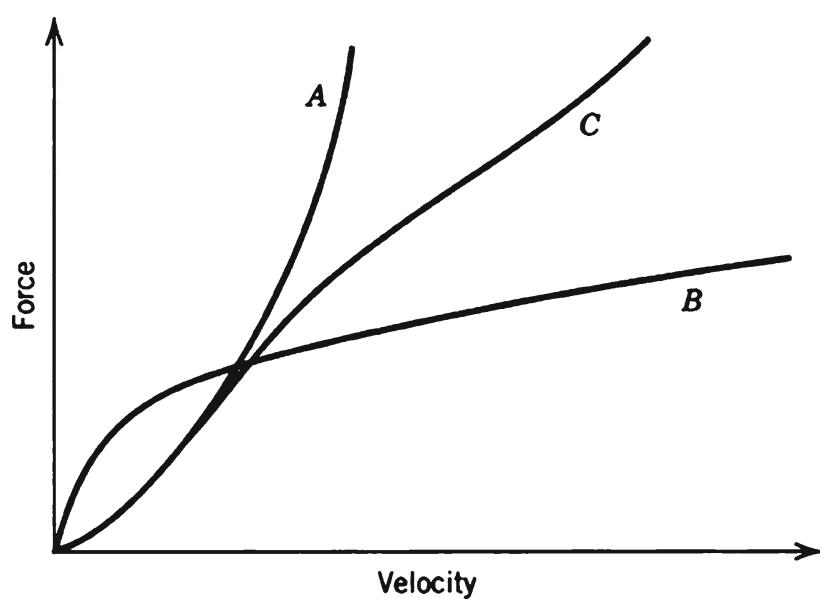

Fig. 4 Damping curves

With the boundary condition of:

$$
B\left(u, \frac{\partial u}{\partial n}\right)=0, \quad r \in \Gamma
$$

where $A$ is a general differential operator, $B$ a boundary operator, $f(r)$ a known analytical function and $\Gamma$ is the boundary of the domain $\Omega$.

$A$ can be divided into two parts which are $L$ and $N$, where $L$ is linear and $N$ is nonlinear. Equation (2) can therefore be rewritten as follows:

$$
L(u)+N(u)-f(r)=0, \quad r \in \Omega,
$$


Homotopy perturbation structure is shown as follows:

$$
H(v, p)=(1-p)\left[L(v)-L\left(u_{0}\right)\right]+p[A(v)-f(r)]=0,
$$

where,

$$
v(r, p): \Omega \times[0,1] \rightarrow R,
$$

In Eq. (5), $p \in[0,1]$ is an embedding parameter and $u_{0}$ is the first approximation that satisfies the boundary condition. We can assume that the solution of Eq. (14) can be written as a power series in $p$, as following:

$$
v=v_{0}+p v_{1}+p^{2} v_{2}+\cdots,
$$

and the best approximation for solution is:

$$
u=\lim _{p \rightarrow 1} v=v_{0}+v_{1}+v_{2}+\cdots,
$$

\section{Enhanced homotopy perturbation method}

Using HPM in some equations may lead to a different or unexpected answer. This problem specifically occurs when a stable nonlinear differential equation has an unstable linear part. This problem has to be treated to match the actual solution. A possible way of treatment is briefly introduced. In this method, the stability property of the nonlinear differential equation will be examined first. If it is provided, the stability of the usual distinguished linear part will be tested. It is hoped that the usual HPM leads to a satisfactory result. If not, there is no guarantee to have a well-behaved result. At this point, an additional zero-term (an extra term with a globally zero effect, i.e., $\eta(x) \in \Omega(x)$ space of linear function with real coefficients) may cope with the problem. At this stage, an extra term will be added to the linear part and subtracted from the rest, at the same time, such that a linear part is stabilized. The rest of the statement establishes the nonlinear part, i.e., $N(v)$. By this alteration and using HPM, a so-called enhanced homotopy perturbation method (EHPM) will produce a stable $v_{0}$, and therefore the whole solutions can be achieved [14].

\section{Applications}

\subsection{Mathematical modeling of shock absorber}

There are many applications where we can derive an expression for the spring force as a function of deflection or the damping force as a function of velocity [1]; in practice forms for the spring relation are

- the linear model: $f(x)=k(x)$

- the cubic model as shown in Eq. (1): $f(x)=k_{1}(x)+k_{2}(x)^{3}$

In which the constant term and the $x^{2}$ term cannot appear if the force function is anti-symmetric about the origin; i.e., if $f(-x)=-f(x)$.

The most common model forms for the damping relation are:

- The linear model: $f(v)=c v$

- The square-law model: $f(v)=c v^{2}$

- The general progressive model (of which the square law is a special case); $f(v)=c v^{n}, n>1$

- The degressive model: $f(v)=c v^{n}, n<1$

The difference between the progressive and the degressive model is that the slope of the progressive curve increase with $v$. The slope of the linear model is constant, and its line between the progressive and the degressive curves (Fig. 4). 


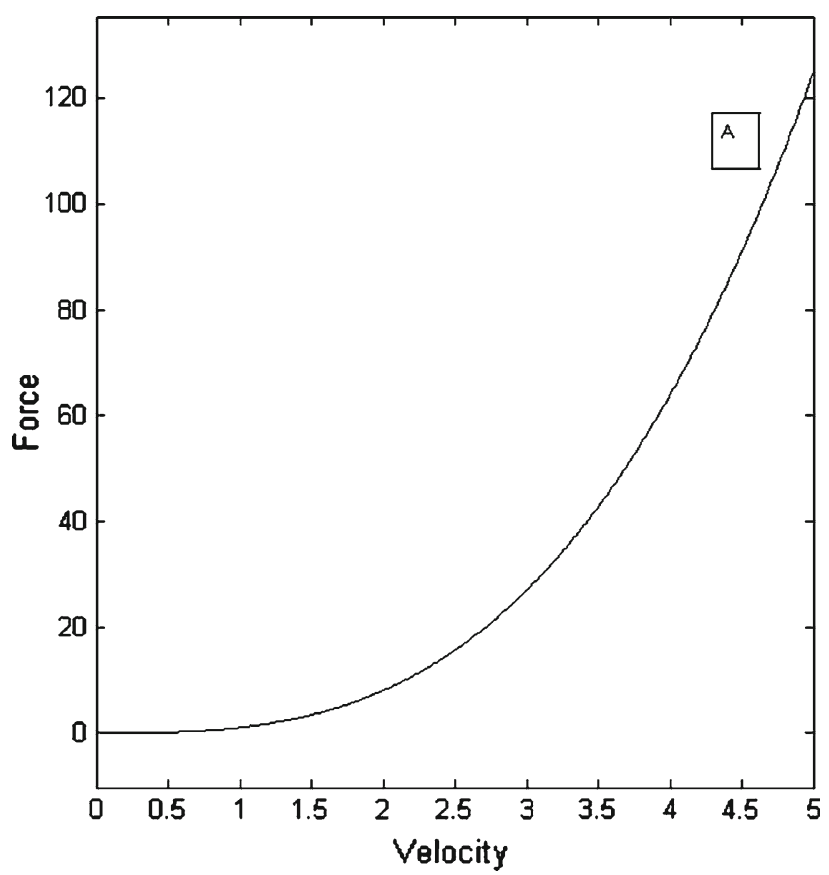

Fig. 5 Progressive nonlinear damping force (N)-versus-velocity (m/s)

\subsection{Case 1}

The shock absorber with a progressive nonlinear damper and linear spring is modeled [1]. The damping force and spring force be described by the following functions, respectively:

$$
F_{d}=v^{3} \text { and } F_{s}=100 x .
$$

where the $v$ is velocity $(\mathrm{m} / \mathrm{s})$ and $x$ is displacement $(\mathrm{m})$.

The damping force-versus-velocity curve is shown in Fig. 5:

We consider the equation of motion and the initial conditions as:

$$
\frac{\mathrm{d}^{2} x}{\mathrm{~d} t^{2}}+\left(\frac{\mathrm{d} x}{\mathrm{~d} t}\right)^{3}+100 x=0, \quad x(0)=0, \quad \frac{\mathrm{d} x}{\mathrm{~d} t}(0)=1,
$$

The linear and the nonlinear parts can be distinguished as:

$$
L(x)=\frac{\mathrm{d}^{2} x}{\mathrm{~d} t^{2}}+100 x
$$

and

$$
N(x)=\left(\frac{\mathrm{d} x}{\mathrm{~d} t}\right)^{3}
$$

By adding and subtracting $\left(a \frac{\mathrm{d}^{2} x}{\mathrm{~d} t^{2}}+b \frac{\mathrm{d} x}{\mathrm{~d} t}\right)$ in Eq. (10) we have:

$$
\begin{aligned}
& \frac{\mathrm{d}^{2} x}{\mathrm{~d} t^{2}}+\left(\frac{\mathrm{d} x}{\mathrm{~d} t}\right)^{3}+100 x+\left(a \frac{\mathrm{d}^{2} x}{\mathrm{~d} t^{2}}+b \frac{\mathrm{d} x}{\mathrm{~d} t}\right)-\left(a \frac{\mathrm{d}^{2} x}{\mathrm{~d} t^{2}}+b \frac{\mathrm{d} x}{\mathrm{~d} t}\right)=0, \\
& x(0)=0, \frac{\mathrm{d} x}{\mathrm{~d} t}(0)=1
\end{aligned}
$$


The new $L(x)$ and $N(x)$ are:

$$
L(x)=\frac{\mathrm{d}^{2} x}{\mathrm{~d} t^{2}}+a \frac{\mathrm{d}^{2} x}{\mathrm{~d} t^{2}}+b \frac{\mathrm{d} x}{\mathrm{~d} t}+100 x
$$

and:

$$
N(x)=\left(\frac{\mathrm{d} x}{\mathrm{~d} t}\right)^{3}-a \frac{\mathrm{d}^{2} x}{\mathrm{~d} t^{2}}-b \frac{\mathrm{d} x}{\mathrm{~d} t},
$$

According to Eq. (5), the homotopy function can be written as:

$$
H(p, x)=(1-p)[L(v)]+p[L(v)+N(v)] .
$$

We choose $a=0.2$ and $b=0.5$. By using $v$ as $v=v_{0}+p v_{1}+p^{2} v_{2}+\cdots$ and substituting into Eq. (16) and rearranging based on powers of $p$-terms, we can obtain:

$$
p^{0}: 1.2 \frac{\mathrm{d}^{2} v_{0}}{\mathrm{~d} t^{2}}+0.5 \frac{\mathrm{d} v_{0}}{\mathrm{~d} t}+100 v_{0}=0, \quad v_{0}(0)=0, \quad \frac{\mathrm{d} v_{0}}{\mathrm{~d} t}(0)=1
$$

and:

$$
\begin{aligned}
& p^{1}: 1.2 \frac{\mathrm{d}^{2} v_{1}}{\mathrm{~d} t^{2}}+0.5 \frac{\mathrm{d} v_{1}}{\mathrm{~d} t}+100 v_{1}+\left(\frac{\mathrm{d} v_{0}}{\mathrm{~d} t}\right)^{3}-0.5 \frac{\mathrm{d} v_{0}}{\mathrm{~d} t}-0.2 \frac{\mathrm{d}^{2} v_{0}}{\mathrm{~d} t^{2}}=0 \\
& v_{1}(0)=0, \quad \frac{\mathrm{d} v_{1}}{\mathrm{~d} t}(0)=0
\end{aligned}
$$

By solving the simple equation in (17), we have the solution as:

$$
v_{0}(t)=0.11 \mathrm{e}^{-0.208 t} \sin (9.11 t) .
$$

The approximation for solution is:

$$
x(t)=\lim _{p \rightarrow 1} v=v_{0}+v_{1}+v_{2}+\cdots,
$$

The first order of EHPM solution is chosen. The solution of the nonlinear shock absorber equation (10), when $p \rightarrow 1$, will be as follows:

$$
x(t)=0.11 \mathrm{e}^{-0.208 t} \sin (9.11 t),
$$

By the drawing figures of numerical solution of Runge-Kutta method and EHPM solution, we see that figure similar to each other (Fig. 6):

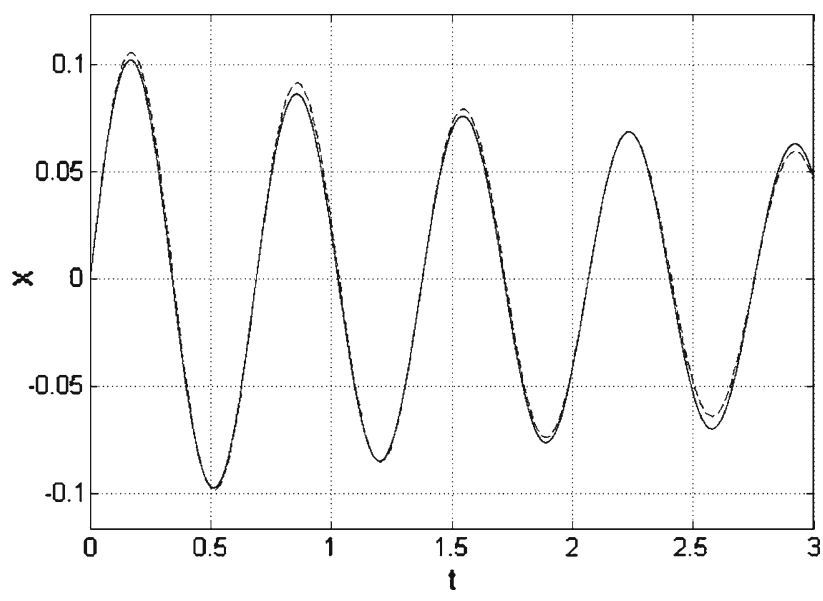

Fig. 6 Comparison of the first order of EHPM solution with the numerical solution of Runge-Kutta method. Dashed line the EHPM solution and solid line numerical solution (displacement (m)-versus-time (s)) 


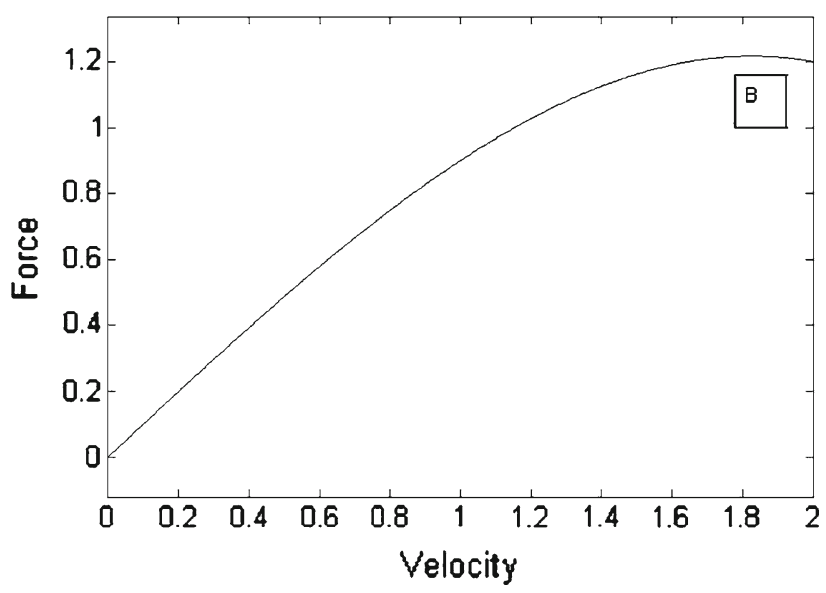

Fig. 7 Degressive nonlinear damping force (force (N)-versus-velocity $(\mathrm{m} / \mathrm{s})$ )

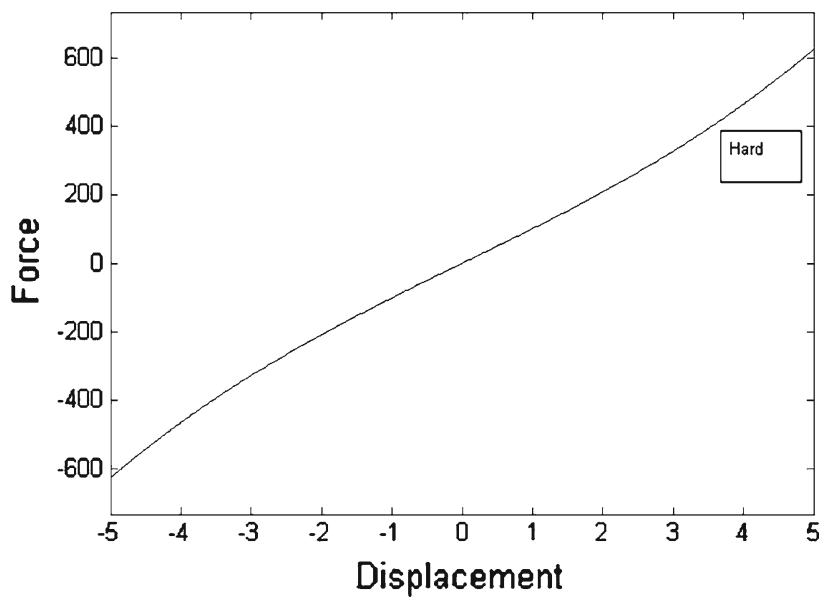

Fig. 8 Hard spring function (force (N)-versus-displacement (m))

\subsection{Case 2}

The shock absorber with a digressive nonlinear damper and nonlinear spring is modeled [1]. The damping force and spring force be described by the following functions, respectively:

$$
F_{d}=v-0.1 v^{3} \text { and } F_{s}=x^{3}+100 x
$$

where the $v$ is velocity $(\mathrm{m} / \mathrm{s})$ and $x$ is displacement $(\mathrm{m})$.

The damping force-versus-velocity curve is shown in Fig. 7:

The spring force-versus-displacement curve is shown in Fig. 8:

The spring element is hard because its slope thus its stiffness increase with deflection.

The equation of motion and the initial conditions are assumed as follows:

$$
\frac{\mathrm{d}^{2} x}{\mathrm{~d} t^{2}}+\frac{\mathrm{d} x}{\mathrm{~d} t}-0.1\left(\frac{\mathrm{d} x}{\mathrm{~d} t}\right)^{3}+x^{3}+100 x=0, \quad x(0)=0, \frac{\mathrm{d} x}{\mathrm{~d} t}(0)=1 .
$$

The linear and the nonlinear parts can be distinguished as:

$$
L(x)=\frac{\mathrm{d}^{2} x}{\mathrm{~d} t^{2}}+\frac{\mathrm{d} x}{\mathrm{~d} t}+100 x
$$

and:

$$
N(x)=-0.1\left(\frac{\mathrm{d} x}{\mathrm{~d} t}\right)^{3}+x^{3}
$$




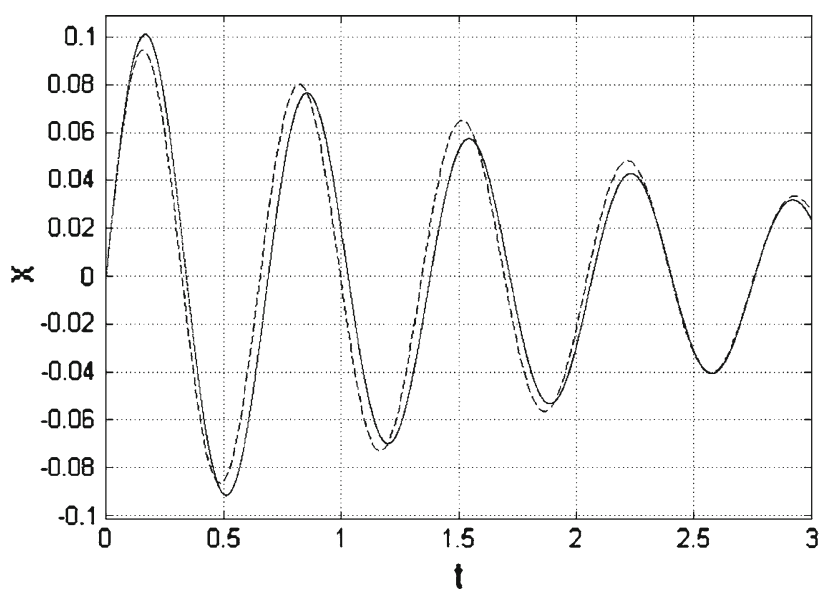

Fig. 9 Comparison of the second order of EHPM solutions of equation of motion (m) with the numerical solution of Runge-Kutta method. Dashed line the EHPM solution and solid line numerical solution

By adding and subtracting $\left(a \frac{\mathrm{d}^{2} x}{\mathrm{~d} t^{2}}+b \frac{\mathrm{d} x}{\mathrm{~d} t}\right)$ in Eq. (23) we have:

$$
\begin{aligned}
& \frac{\mathrm{d}^{2} x}{\mathrm{~d} t^{2}}+\frac{\mathrm{d} x}{\mathrm{~d} t}-0.1\left(\frac{\mathrm{d} x}{\mathrm{~d} t}\right)^{3}+x^{3}+100 x+\left(a \frac{\mathrm{d}^{2} x}{\mathrm{~d} t^{2}}+b \frac{\mathrm{d} x}{\mathrm{~d} t}\right)-\left(a \frac{\mathrm{d}^{2} x}{\mathrm{~d} t^{2}}+b \frac{\mathrm{d} x}{\mathrm{~d} t}\right)=0, \\
& x(0)=0, \frac{\mathrm{d} x}{\mathrm{~d} t}(0)=1 .
\end{aligned}
$$

The new $L(x)$ and $N(x)$ are:

$$
L(x)=\frac{\mathrm{d}^{2} x}{\mathrm{~d} t^{2}}+\frac{\mathrm{d} x}{\mathrm{~d} t}+100 x+\left(a \frac{\mathrm{d}^{2} x}{\mathrm{~d} t^{2}}+b \frac{\mathrm{d} x}{\mathrm{~d} t}\right)
$$

and:

$$
N(x)=-0.1\left(\frac{\mathrm{d} x}{\mathrm{~d} t}\right)^{3}+x^{3}-\left(a \frac{\mathrm{d}^{2} x}{\mathrm{~d} t^{2}}+b \frac{\mathrm{d} x}{\mathrm{~d} t}\right)
$$

According to Eq. (5), the homotopy function can be written as similar as Eq. (16):

$$
H(p, x)=(1-p)[L(v)]+p[L(v)+N(v)],
$$

We consider $a=0.3$ and $b=1.2$. By using $v$ as $v=v_{0}+p v_{1}+p^{2} v_{2}+\cdots$ and substituting $v$ into Eq. (29) and rearranging based on powers of $p$-terms, we can obtain:

$$
p^{0}: 1.3 \frac{\mathrm{d}^{2} v_{0}}{\mathrm{~d} t^{2}}+2.2 \frac{\mathrm{d} v_{0}}{\mathrm{~d} t}+100 v_{0}=0, \quad v_{0}(0)=0, \quad \frac{\mathrm{d} v_{0}}{\mathrm{~d} t}(0)=1,
$$

and:

$$
\begin{aligned}
& p^{1}: 1.3 \frac{\mathrm{d}^{2} v_{1}}{\mathrm{~d} t^{2}}+2.2 \frac{\mathrm{d} v_{1}}{\mathrm{~d} t}+100 v_{1}-0.1\left(\frac{\mathrm{d} v_{0}}{\mathrm{~d} t}\right)^{3}-0.3 \frac{\mathrm{d}^{2} v_{0}}{\mathrm{~d} t^{2}}-1.2 \frac{\mathrm{d} v_{0}}{\mathrm{~d} t}+v_{0}^{3}=0, \\
& v_{1}(0)=0, \quad \frac{\mathrm{d} v_{1}}{\mathrm{~d} t}(0)=0,
\end{aligned}
$$

By solving the simple equations in (30), (31) we have the solution as:

$$
v_{0}(t)=0.114 \mathrm{e}^{-0.846 t} \sin (8.72 t)
$$


and:

$$
\begin{aligned}
v_{1}(t)= & -0.0049 \mathrm{e}^{-0.846 t} \sin (8.72 t)-0.0017 \mathrm{e}^{-0.0846 t} \cos (8.72 t) \\
& +6.45 \times 10^{-22}\left[\left(1.85 \times 10^{20} t+2.71 \times 10^{18}\right) \mathrm{e}^{-2.54 t} \mathrm{e}^{1.69 t}-4.51 \times 10^{16} \mathrm{e}^{-2.54 t}\right] \cos (8.72 t) \\
& +0.0305\left[-0.0647+\left(-0.224+t \mathrm{e}^{1.69 t}\right)\right] \mathrm{e}^{-2.54 t} \sin (8.72 t) \\
& +6.45 \times 10^{-22}\left[6.39 \times 10^{15} \sin (26.2 t)-4.87 \times 10^{16} \cos (26.2 t)\right] \mathrm{e}^{-2.54 t}
\end{aligned}
$$

The approximation for solution is:

$$
x(t)=\lim _{p \rightarrow 1} v=v_{0}+v_{1}+v_{2}+\cdots,
$$

The second-order EHPM is chosen. The solution of the nonlinear shock absorber equation (32), when $p \rightarrow 1$, will be as follows:

$$
x(t)=v_{0}+v_{1} .
$$

By the figures of numerical solution of Runge-Kutta method and EHPM solution, we see that the figures are similar to each other.

\section{Conclusions}

The EHPM was applied to nonlinear shock absorber and spring equations. The method which is proved to be a powerful mathematical tool for studying nonlinear equations can be easily extended to any nonlinear equation. This method is based on stabilizing the unstable linear part by adding and subtracting an extra linear term.

Moreover, we showed that the obtained solutions are valid for the whole domain. We show that even the lowest order approximations obtained by the present theory are actually of high accuracy.

Open Access This article is distributed under the terms of the Creative Commons Attribution Noncommercial License which permits any noncommercial use, distribution, and reproduction in any medium, provided the original author(s) and source are credited.

\section{References}

1. Palm, W.J.: Mechanical vibration, vol. 1. ISBN 0-471-34555-5, 9

2. Vidyasagar, M.: Nonlinear systems analysis, 2nd edn. Prentice-Hall, Englewood Cliffs, ISBN 0-13-623513-1 (1993)

3. Khalil, H.: Nonlinear systems, vol. 2. ISBN 964-6213-22-7, 8

4. He, J.H.: The homotopy perturbation method for nonlinear oscillators with discontinuities. Appl. Math. Comput. 151(1), 287292 (2004)

5. Ganji, D.D.: The application of He's homotopy perturbation method to nonlinear equation arising in heat transfer. Phys. Lett. A 355, 337-341 (2006)

6. Ganji, D.D., Rajabi, A.: Assessment of homotopy perturbation and perturbation method in heat radiation equations. Int. Commun. Heat Mass Transf. 33(3), 391-400 (2006)

7. He, J.H.: Homotopy perturbation method for solving boundary value problems. Phys. Lett. A 350(1-2), 87-88 (2006)

8. He, J.H.: Application of homotopy perturbation method to nonlinear wave equations. Chaos Solut. Fractals 26(3), 695$700(2005)$

9. He, J.H.: Homotopy perturbation method for bifurcation of nonlinear problems. Int. J. Nonlinear Sci. Numer. Simul. 6(2), 207-208 (2005)

10. Ganji, D.D.: The application of He's homotopy perturbation method to nonlinear equations arising in heat transfer. Phys. Lett. A 355(4-5), 337-341 (2006)

11. Ganji, D.D.: The application of He's homotopy perturbation method to nonlinear equation arising in heat transfer. Phys. Lett. A 355, 337-341 (2006)

12. Ganji, D.D., Rajabi, A.: Assessment of homotopy perturbation and perturbation method in heat radiation equations. Int. Commun. Heat Mass Transf. 33(3), 391-400 (2006)

13. Adomian, G.: A review of the decomposition method in applied mathematics. Math. Anal. Appl. 135, 501-544 (1988)

14. Hosein Nia, S.H., Soltani, H., Ghasemi, J., Ranjbar, A.N., Ganji, D.D.: Maintaining the stability of nonlinear differential equations by the enhancement of HPM. Phys. Lett. A 372(16), 2855-2861 (2008) 